\title{
DETERMINACIÓN COPROLÓGICA DE LA PARASITOFAUNA EN CERDOS CRIOLLOS (Sus scrofa domestica) EN EL DEPARTAMENTO DE CÓRDOBA, COLOMBIA
}

\section{COPROLOGICAL PARASITEFAUNA DETERMINATION OF PIGS IN CREOLE (Sus scrofa domestica) IN THE DEPARTMENT OF CORDOBA, COLOMBIA}

\author{
YONAIRO HERRERA B,${ }^{1 *} \mathrm{M} . S c(c)$, MICHAEL ALMANZA P, ${ }^{1,2} \mathrm{MVZ}$, CARLOS ENSUNCHO H, ${ }^{1} \mathrm{MVZ}$, \\ LUIS GOMÉZ M, ${ }^{1,2}$ MVZ, MARCOS GALEANO E,, ${ }^{1,2} \mathrm{MVZ}$.
}

${ }^{1}$ Universidad de Córdoba. Programa de Medicina Veterinaria y Zootecnia. Departamento de Ciencias Pecuarias. Montería. Colombia. ${ }^{2}$ Grupo de Investigación en Parasitología y Salud Publica - REDIA.

\section{Key words:}

Gastrointestinal parasites, nematodes, cestodes, protozoa.

\section{Abstract}

The objective of the research was to determine the parasitic fauna in Colombian creole pigs reared outdoors in the department of Cordoba, Colombia. The study was conducted on 62 farms, examining a total of 166 samples using the technique of flotation and McMaster. The found results demonstrate the presence of 10 genera of nematodes, one of protozoa and one of acanthocephalan. It is to emphasize the absence of tapeworms and flukes in the study. Regarding the age of the animals, increased frequency of gastrointestinal parasites in young animals relative to adult animals was found. These infections by coccidia and nematodes can be considered as a problem of medical and economic importance should be subject to greater attention for its control since they act as constraints on production systems, being associated to losses and delays in production and profitability herds.

\section{Palabras Clave:}

Parásitos gastrointestinales, nemátodos, céstodos, protozoos.

\section{INFORMACIÓN}

Recibido: 05-07-2015;

Aceptado: $26-10-2015$

Correspondencia autor:

yonairo@yahoo.es

\section{Resumen}

El objetivo de la investigación fue determinar la fauna parasitaria en Cerdos Criollos Colombianos criados al aire libre en el departamento de Córdoba, Colombia. El estudio se llevó a cabo en 62 predios, examinando un total de 166 muestras haciendo uso de la técnica de Flotación. Los resultados hallados demuestran la presencia de 10 géneros de nematodos, uno de protozoos y uno de acantocéfalo. Se ha de destacar la ausencia de cestodos y trematodos en el estudio. En relación a la edad de los animales, se encontró mayor frecuencia de parásitos gastrointestinales en animales jóvenes en relación con los animales adultos. Estas infecciones por coccidias y nematodos pueden considerarse como un problema de importancia médica y económica que debe ser objeto de mayor atención para su control ya que actúan como limitantes en los sistemas de producción, siendo asociadas a pérdidas y retrasos en la producción y rentabilidad de las piaras. 


\section{Introducción}

Las parasitosis gastrointestinales son generalmente producidas por helmintos (nemátodos, céstodos) y protozoarios. Representan una amenaza para los animales domésticos, ya que causan anorexia, reducción en la ingestión de alimentos, disminución en la conversión alimenticia, pérdidas de sangre y de proteínas plasmáticas en el tracto gastrointestinal, alteraciones en el metabolismo proteico, reducción de minerales, depresión en la actividad de algunas enzimas intestinales y diarrea (RODRÍGUEZ et al., 2001). Contribuyendo de esta forma en la presentación de enfermedades concomitantes, aumento en los costos de producción, gastos en medicamentos y servicios veterinarios, afectando la rentabilidad de la producción pecuaria por decomiso de órganos (QUIJADA, 2010). Además de los problemas económicos que generan las infestaciones e infecciones parasitarias, es importante destacar el riesgo que representan para la salud humana las infecciones zoonóticas causadas por parásitos como Taenia solium y Triquinella spp, esta última no reportada para Colombia (VALLE et al., 2006)

En las explotaciones al aire libre existe una limitación en el ámbito de la quimio-prevención que hace que los animales tengan acceso a hospedadores intermediarios o paraténicos y se incrementa el contacto con las heces o cadáveres de animales domésticos y silvestres infectados, lo que favorece una mayor transmisión de los parásitos, tanto de ciclo directo como indirecto (PERRY, 1999).

La porcicultura se ha enfocado en satisfacer las necesidades comerciales, con nuevas técnicas de manejo y mejoras genéticas de alta eficiencia productiva. Las razas criollas tienen un mercado menor y se ha olvidado la importancia que tiene el cerdo criollo colombiano en los sistemas de producción, con sus aportes en características de supervivencia, adaptabilidad, independencia al momento de la cría, resistencia a las enfermedades, rusticidad, capacidad transformadora y como reservorio de genes que pueden mejorar cualidades en producción y reproducción (LÓPEZ et al., 2014).

Realizar el diagnóstico y determinar la frecuencia de los mismos en las poblaciones de animales es primordial para el diseño de programas de manejo integral que permitan preservar la población refugio e intervenir en los animales con cargas parasitarias que afectan la salud, la productividad y el bienestar animal. Es por ello que el objetivo principal de esta investigación fue la determinación de la fauna parasitaria en cerdos criollos colombianos criados al aire libre en el departamento de Córdoba.

\section{Materiales y método}

Área de estudio: El estudio se llevó a cabo en piaras ubicadas en el departamento de Córdoba, Colombia, ubicado entre las coordenadas $7^{\circ} 23^{\prime}$ y $9^{\circ} 26^{\prime}$ de latitud norte y los $74^{\circ} 52^{\prime}$ y $76^{\circ} 32^{\prime}$ de longitud oeste, a una altura de 30 m.s.n.m, con temperatura promedio anual de 28 ${ }^{\circ} \mathrm{C}$, humedad relativa del $84 \%$, precipitación media anual de $1200 \mathrm{~mm}$ y pertenece a la formación climática de bosque tropical lluvioso (CASSAB et al., 2010)

Animales de estudio: Para esta investigación se trabajó con Cerdos Criollos (Sus scrofa domestica) clínicamente sanos con el fin de determinar la fauna parasitaria gastrointestinal. Los animales fueron clasificados por sexo y edad (jóvenes y adultos). Todos los animales de esta investigación eran mantenidos la mayor parte del tiempo al aire libre. El estudio fue de tipo descriptivo transversal y se llevó a cabo durante los meses de abril, mayo y junio de 2015. El tamaño de la muestra fue por conveniencia y se procesaron 166 muestras de heces de 166 animales las cuales fueron tomadas en 62 predios campesinos del departamento.

Métodos parasitológicos: Las muestras fueron recolectadas directamente del recto del animal y de forma no invasiva de heces recién defecadas. Luego fueron depositadas en frascos estériles recolectores de materia fecal, debidamente rotulados con especie, edad y sexo del animal. Las muestras recolectadas fueron almacenadas en cavas de icopor $\circledast$ con balas congeladas, evitando al máximo la exposición a factores externos como calor y corrientes de aire que pudieran ocasionar daños a las muestras, las cuales se transportaron al laboratorio de Parasitología de la Facultad de Medicina Veterinaria y Zootecnia de la Universidad de Córdoba, en un tiempo menor a doce horas, donde se procedió al análisis de las mismas. La técnica utilizada fue flotación (RODRÍGUEZ, 2005).

Análisis estadístico: Los resultados de esta investigación fueron tabulados en Microsoft Excel versión 2010 y analizados por el programa estadístico InfoStat e2015, estimando la frecuencia mediante la fórmula descrita por ALTMAN y BLAND (2001): Frecuencia $=($ No. Animales positivos $/$ Población total $)$ x 100 .

\section{Resultados}

De acuerdo a los resultados obtenidos, de las 166 muestras examinadas, el 97,5\% (162) resultó positivo al menos para algún grupo parasitario. La fauna encontrada para protozoos y nematodos se muestra en la Tabla 1. Del total de muestras, el grupo parasitario que mayores frecuencias obtuvo fue el de protozoos 
(Eimeria spp) con 121 animales positivos (72,89\%). De la misma manera la Tabla 1, evidencia la presencia de 12 géneros de parásitos en el cerdo criollo colombiano.

Tabla 1. Frecuencia general de parásitos gastrointestinales en cerdos criollos colombiano.

\begin{tabular}{lcc}
\hline \multicolumn{1}{c}{ Género } & Positivos (n) & Frecuencia (\%) \\
\hline Eimeria spp. & 121 & 72,89 \\
Strongyloides spp. & 84 & 50,6 \\
Trichostrongylus spp. & 74 & 44,58 \\
Oesophagostomun spp. & 32 & 19,28 \\
Tyroglyphus & 25 & 15,06 \\
Globocephalus & 13 & 7,83 \\
Ascaris suum & 22 & 13,25 \\
Metastrongylus apri & 9 & 5,42 \\
Hyostrongylus rubidus & 4 & 2,41 \\
Ascarops dentata & 22 & 13.25 \\
Trichuris suis & 2 & 1,2 \\
Macracanthorhynchus hirudinaceus & 2 & 1,2 \\
\hline
\end{tabular}

En la tabla 2 se observan las frecuencias parasitarias de acuerdo a la edad, animales jóvenes ( $<4$ meses) y animales adultos ( $\geq 4$ meses).

Tabla 2. Frecuencia por edad de parásitos gastrointestinales en cerdos criollos colombiano.

\begin{tabular}{lcc}
\hline \multicolumn{1}{c}{ Parásito } & Frecuencia jóvenes (\%) & Frecuencia adultos (\%) \\
\hline Eimeria spp. & 80,77 & 65,71 \\
Trichostrongylus spp. & 73,08 & 40 \\
Strongyloides & 57,69 & 71,43 \\
Oesophagostomum & 38,46 & 11,43 \\
dentatum & 19,23 & 14,29 \\
Tyroglyphus & 15,38 & 2,86 \\
Metastrongylus apri & 7,69 & 14,29 \\
Ascaris suum & 3,85 & 5,71 \\
Globocephalus & 3,85 & 2,86 \\
Ascarops Dentata & 0 & 11,43 \\
Hyostrongylus rubidus & 0 & 2,86 \\
Macracanthorhynchus & & \\
hirudinaceus & &
\end{tabular}

En la tabla 3, se detalla la clasificación de acuerdo a las asociaciones parasitarias.

Tabla 3. Porcentaje de presentación de las distintas asociaciones parasitarias.

\begin{tabular}{ccc}
\hline Taxones & Cerdos parasitados & $\% 1$ \\
\hline Monoparasitismo & 32 & 19,75 \\
Biparasitismo & 65 & 40,12 \\
Triparasitismo & 38 & 23,46 \\
Tetraparasitismo & 20 & 12,35 \\
Pentaparasitismo & 7 & 4,32 \\
\hline
\end{tabular}

${ }^{1}$ En base a 162 cerdos parasitados

\section{Discusión}

En cuanto a las especies y géneros hallados y según grupos taxonómicos, se han detectado 10 géneros de nematodos, uno de protozoos y uno de acantocéfalos. Se ha de destacar por otra parte la ausencia de cestodos y trematodos en el estudio.

En el grupo de nematodos el género que presento mayores positividades fue Strongyloides con un $50,6 \%$ (84 muestras positivas), seguido del género Trichostrongylus spp y Oesophagostomun spp, con un $44,58 \%$ (74) y $19,28 \%$ (32), respectivamente.

La localización de los géneros encontrados en los coprológicos son en su mayoría el tubo digestivo, así vemos como en el grupo de protozoos, las Eimerias se sitúan a nivel de intestino delgado; en el grupo de nematodos Ascarops en estómago, Ascaris, Globocephalus, Hyostrongylus, Trichostrongylus y Strongyloides en intestino delgado, Oesophagostomun y Trichuris se ubican en intestino grueso; Metastrongylus se encuentra en vías respiratorias, y finalmente el único acantocéfalo evidenciado, Macracanthorhynchus hirudinaceus se localiza en intestino delgado.

La fauna encontrada en la investigación es amplia en comparación con el estudio realizado en el corregimiento de San Roque, Antioquia, en el que se observaron solamente huevos de Strongyloides spp., Strongylidos spp., Trichuris spp., Ascaris suum y coccidias. (ARIAS, et al., 2014). LUNA y KYVSGAARD (2005) en Nicaragua observaron que las especies encontradas fueron Ascaris suum, Hyostrongilus rubidus, Strongyloides ransomi, Oesophagostomun spp., Trichuris suis, Isospora suis, Eimeria spp. y Metastrongylus spp.

Es importante tener presente que la parasitosis porcina tiene fuerte relación con el hábitat y el sistema de explotación donde son manejados los animales. Desde el punto de vista parasitológico el cerdo puede estar infestado por protozoos, helmintos y artrópodos (CASTILLO et al., 2001).

La coccidiosis en cerdos domésticos generalmente es un problema asociado al confinamiento (MUNDIM y et al., 2004; GARCÍA, 1999), así como también se asocia a otros parásitos especialmente a nematodos gastrointestinales, y en menor proporción a nematodos broncopulmonares, y otras parasitosis o agentes infecciosos (RESPALDIZA, 2007).

GARCÍA (1999), agrega que las condiciones ambientales como alta humedad, temperaturas medias y acumulo de heces facilitan la supervivencia de los ooquistes, y por 
lo tanto, el contagio, condiciones que efectivamente se presentan en el departamento de Córdoba. HENRY y TOKACH (1995), y VALLE et al., (2006), mencionan la relación entre las explotaciones con bajo nivel sanitario y la presentación de ooquistes en el suelo, o adheridos a las mamas de las hembras.

La presencia de Eimeria spp en cerdos es considerada por algunos autores como un indicador del nivel de higiene del lugar en donde se encuentran los animales, la presencia de Eimeria spp está relacionado con un bajo nivel de higiene (KARAMON et al., 2007). Además, presentan un corto período de prepatencia, propiciando una infección y propagación lateral muy rápida (VALLE et al., 2006).

En Colombia son pocos los estudios realizados sobre parasitismo gastrointestinal en la especie porcina, especialmente en los cerdos domésticos de sistemas de producción al aire libre. En la presente investigación los exámenes coproparasitarios evidenciaron la constante presencia de ooquistes de coccidios y huevos de nematodos, lo que podría deberse al tipo de explotación y de crianza al aire libre que se presenta en el Departamento, presentándose mayores dificultades en el control del parasitismo, hecho este que favorece la ingestión por parte de los animales de todo tipo de hospedadores intermediarios (lombrices de tierra, caracoles, escarabajos, vegetales silvestres con larvas enquistadas, entre otros); sumado a esto, el sistema de producción permite la alimentación con pastos contaminados, facilitando la infección por ingestión de heces o cadáveres de animales silvestres infectados, ayudando esto a una mayor transmisión, tanto de parásitos de ciclo de vida directo e indirecto.

Es de anotar que a pesar de la escasa signología clínica que presentan la mayoría de las parasitosis en los cerdos, estas representan unos de los principales problemas que afectan el rendimiento productivo de estas especies en traspatio, a la vez que coadyuvan a otras patologías secundarias e influyen directamente en la salud de los animales, y por ende en la producción de carne y fundamentalmente en la economía familiar.

En relación a la edad de los animales la Tabla 2, muestra las frecuencias encontradas en animales jóvenes $(<4$ meses) y animales adultos ( $\geq 4$ meses). Se observa como la frecuencia es superior en animales jóvenes en relación con los animales adultos, a excepción del género Strongyloides, que presentó mayores frecuencias en adultos. La diferencia en la carga de parásitos entre animales menores de un año, y los animales mayores de un año se explica por el desarrollo de la inmunidad por los animales mayores como resultado de las repetidas exposiciones a la infección (HEISE-PAVLOV y HEISE-PAVLOV, 2004; RAJKOVIĆ-JANJE et al., 2002; HUMBERT y HENRY, 1989). A este respecto ALCAIDE et al., (2006), señala que debido al reiterado contacto con el parásito no hay reimplantación de nuevos parásitos en las reinfecciones eliminándose las infecciones existentes. Por su parte, ALDAZ (2003) afirma que los cerdos jóvenes son más susceptibles que los adultos a la infección por los diferentes grupos taxonómicos de parásitos, y que esto se debe al bajo nivel inmunitario que presentan los lechones en las primeras semanas de vida, lo que los lleva a presentar en ocasiones síntomas clínicos incluso con infestaciones leves, además a esta edad los animales no han estado expuestos anteriormente a cargas parasitarias para crear resistencia a los mismos, así como que las infestaciones tempranas por nematodos en cerdos recién nacidos, hace que desarrollen resistencia en poco tiempo.

En cuanto a las asociaciones parasitarias, la asociación más frecuente es la producida por Eimeria spp. y Trichostrongylus spp. con 56 muestras positivas. El biparasitismo es la asociación más frecuente $(40.13 \%)$, seguida del triparasitismo $(23,46 \%)$ y tetraparasitismo $(12,35 \%)$. En solo 7 muestras se obtuvo pentaparasitismo con un 4,32\% (Tabla 3). Este tipo de asociaciones encontradas quizás sean debidas al sistema de crianza al aire libre del cerdo criollo en el departamento, donde todas las categorías animales pastan de manera conjunta, no existiendo sistemas de rotación de potreros según la categoría animal, encontrándose todos los cerdos expuestos a las infecciones por coccidios y nematodos. De igual manera en un huésped porcino, es común la presencia de infecciones parasitarias mixtas, mientras que las infecciones monoespecificas son menos frecuentes y se registran en animales bajo control parasitario intensivo.

\section{Conclusión}

Existe una alta carga parasitaria en los cerdos criollos en el departamento de Córdoba, determinándose la presencia de protozoarios del género Eimeria y nematodos, principalmente. Estas infecciones por coccidias y nematodos pueden considerarse como un problema de importancia médica y económica que debe ser objeto de mayor atención para su control, ya que actúan como limitantes en los sistemas de producción, siendo asociadas a pérdidas y retrasos en la producción y rentabilidad de las piaras. Es importante resaltar que se hace necesaria la creación de planes antiparasitarios especiales para este tipo de explotaciones de campo, de las cuales viven muchos campesinos como sustento económico para sus familias. 


\section{Referencias}

ALCAIDE, M.; FRONTERA, E.; REINA, D. 2006. La importancia de la metastrongylosis porcina en el suroeste español. Una parasitosis en auge. Prod Animal 226:4-15.

ALDAZ, A. 2003. ¿Tienen que convivir los reproductores y los parásitos? Anaporc 23:20-43.

ALTMAN, D.G.; BLAND, J.M. 2001. Statistics Notes: Diagnostic tests 1: sensitivity and specificity. Brit. Med. J 308:1552-1553.

GARCÍA, T. 1999. Endoparasitosis del porcino Ibérico en Extremadura, España: Epidemiología y control. Tesis doctoral. Facultad de Veterinaria. Universidad de Extremadura. España.

CASSAB, A.; MORALES, V. y MATTAR, S. 2010. Factores climáticos y casos de Dengue en Montería, Colombia. 2003-2008. Rev. salud pública 13(1): 115-128.

HEISE-PAVLOV, P.; HEISE-PAVLOV, S. 2004. Prevalence of selected parasites and leptospira in feral pigs (Sus domesticus) from tropical North-Eastern Australia. Galemys 16:211-220.

HENRY. S.; TOKACH, L. 1995. Eimeria-associated pathology in breeding gilts swine. Swine Health in Production 3:200-201.

HUMBERT, J.; HENRY, C. 1989. Studies on the prevalence and the transmission of lung and stomach nematodes of the wild boar (Sus scrofa) in France. J of Wildlife Dis 25:335-341.

KARAMON, J.; ZIOMKO, I.; CENCEK, T. 2007. Prevalence of Isospora suis and Eimeria spp. in suckling piglets and sows in Poland. Vet Parasitol 147:171-175.

LÓPEZ ARIAS, A.; CHAPARRO GUTIÉRREZ, J.; MEJÍA JARAMILLO J.; MORENO, F. 2014. Dinámica parasitaria en cerdo criollo colombiano San Pedreño en condiciones de pastoreo en el trópico. Asociación Colombiana de Porcicultores. Fondo Nacional de la Porcicultura. Febrero-Marzo 2014186 3(2):31-36.

MUNDIM, M.; MUNDIM, A.; SANTOS, A.; CABRAL, D. 2004. Helmintos e protozoários em fezes de javalis (Sus scrofa scrofa) criados em cativeiro. Arq Bras Med Vet Zootec 56:792-795.

PERRY, B.D.; RANDOLPH, T.F. 1999. Improving the assessment of the economic impact of parasitic diseases and of their control in production animals. Vet Parasitology 84:145-168.

QUIJADA, J. 2005. Principales endoparasitosis en porcino según el sistema de producción.. Disponible en: http:// www.Albeitar.grupoasis.co.m/bibliografias/endoparasitosporcinos133.doc Consultada: 18-05-2015.

RAJKOVIĆ-JANJE, R.; BOSNIĆ, S.; ZAGREB. 2002. Prevalence of helminths in wild boars from hunting grounds in eastern Croatia. Z Jagdwiss 48:261-270.

RESPALDIZA, E. 2007. El Jabalí Sus scrofa Linnaeus (1758). Consideraciones epizootiológicas sobre algunas parasitosis y técnicas diagnósticas para su control. Conferencia en la Real Academia de Ciencias Veterinarias. España.

RODRÍGUEZ, R.I.; COB, L.A. 2005. Técnicas diagnósticas en parasitología veterinaria. 2ed. Ediciones de la Universidad Autónoma de Yucatán. Yucatán, México.

RODRÍGUEZ, R.; COB, L.; DOMÍNGUEZ, J. 2015. Frecuencia de parásitos gastrointestinales en animales domésticos diagnosticados en Yucatán, México. Disponible en: http://wwwvady.mx/biomedic/reubiomec/pvf/rb0112/4pdf. Consultada: 22-05-2015.

VALLE, Y.; GUERRA, Y.; MENCHO, J.D.; VÁSQUEZ, A. 2006. Comparación del parasitismo gastrointestinal en cerdos estatales y privados en diferentes categorías. Rev. prod. Anim 18(2):141-144. 